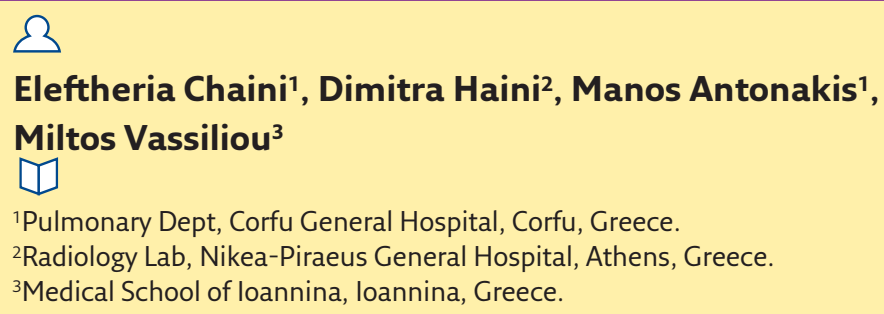

\title{
Severe fever and dyspnoea in a young girl with Hodgkin lymphoma
}

\section{Case report}

A 16-year-old girl was admitted to our department with fever and dyspnoea at rest. Symptoms had started $48 \mathrm{~h}$ earlier, without any beneficial effect of orally given cefuroxime tablets $(500 \mathrm{mg}$ every $12 \mathrm{~h}$ ) during the last 2 days. A Hodgkin lymphoma was diagnosed 5 months before and the currently applied therapeutic schedule included bleomycin (accumulative dose of 90 units). The last applied regimen was given 10 days earlier. Propranolol (Inderal) was given orally for concomitant tachycardia episodes.

The patient's clinical signs included pyrexia $\left(39.5^{\circ} \mathrm{C}\right)$, tachypnoea (22 breaths. $\left.\mathrm{min}^{-1}\right)$, hypoxaemia (pulse oximetry $92 \%$ on room air) and tachycardia (140 beats. $\mathrm{min}^{-1}$ ). Blood pressure was $110 / 70 \mathrm{mmHg}$. Lung auscultation revealed decreased breath sounds at left lung base and fine bibasilar crackles, more prominent at the left side of the thorax. The rest of clinical examination was unremarkable.

The patient underwent a chest radiograph (figure 1).

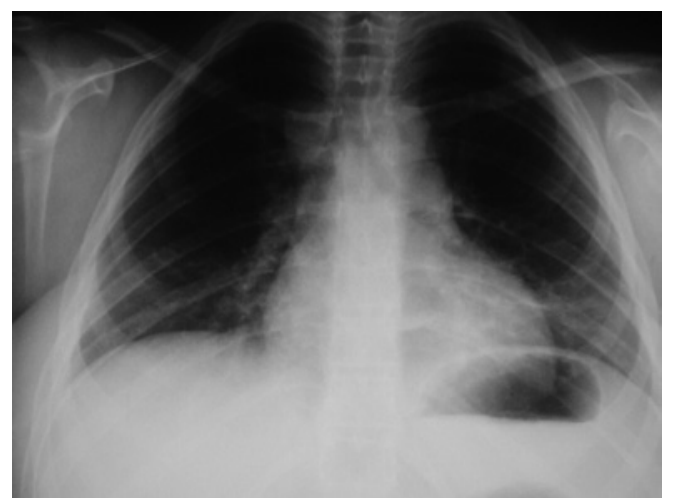

Figure 1 Chest radiograph on admission day.

Task 1

Describe the radiographical findings.
Cite as: Chaini E, Haini D, Antonakis M, et al. Severe fever and dyspnoea in a young girl with Hodgkin lymphoma. Breathe 2017; 13: e10-e16. 


\section{Answer 1}

The chest radiograph showed patchy bibasilar consolidations worse on the left.
Task 2

What is your differential diagnosis? 


\section{Answer 2}

Community-acquired pneumonia (CAP) and acute interstitial pneumonitis due to bleomycin-induced pulmonary toxicity.
Two different diagnoses were considered for this case: CAP and acute interstitial pneumonitis due to bleomycin-induced pulmonary toxicity.

Task 3

What investigation would be helpful? 


\section{Answer 3}

Blood tests, sputum and blood cultures, and urinary antigen tests for Pneumococcus and Legionella.

a)
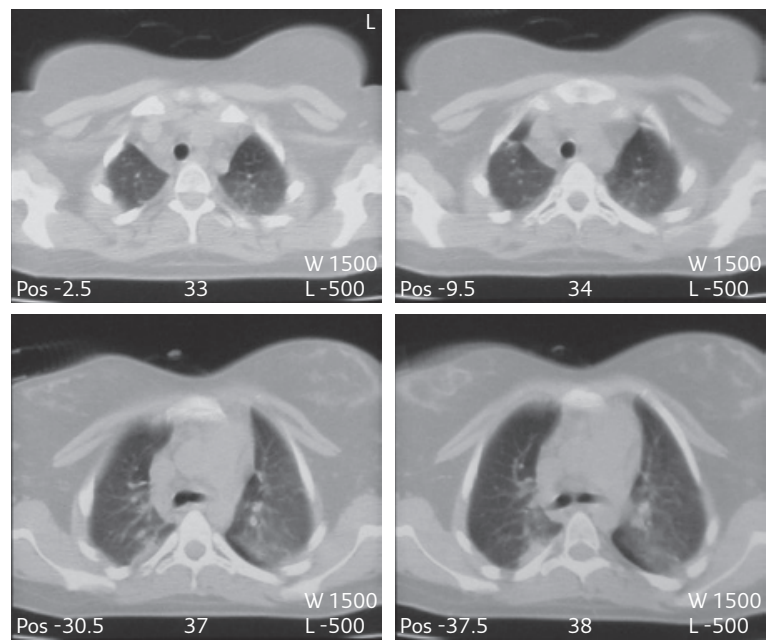

b)
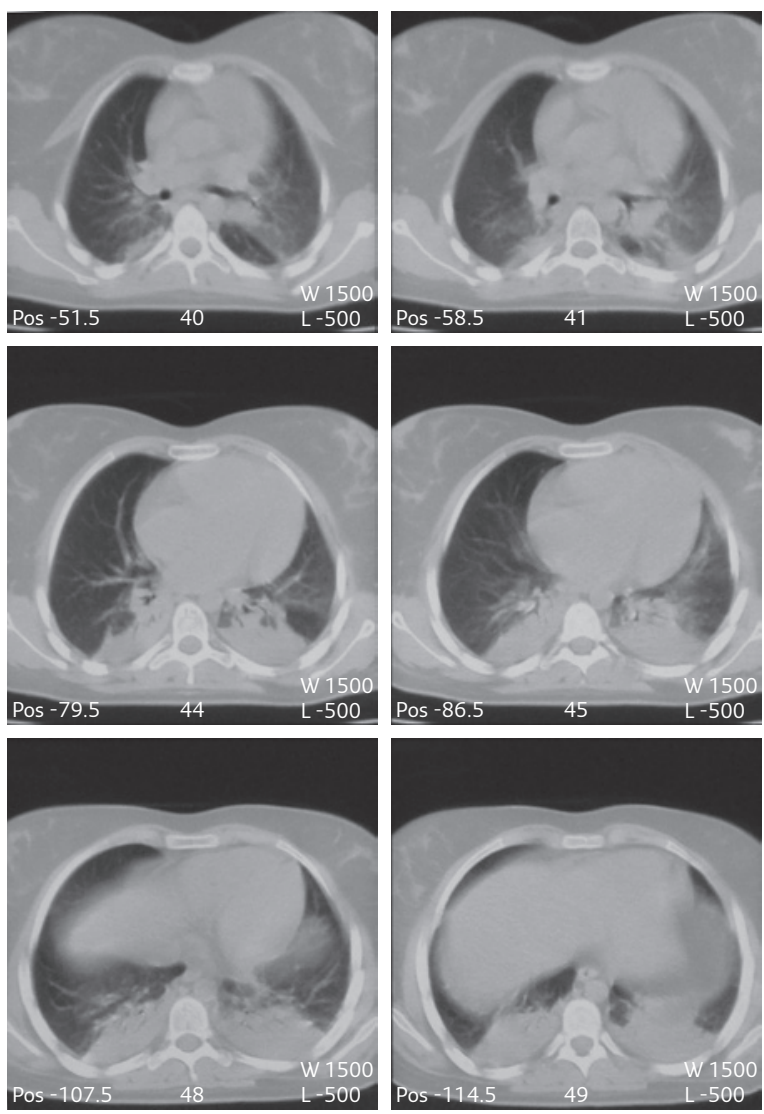

Figure 2 Chest CT on the third day of hospitalisation. a) Upper lung fields, b) middle and lower lung fields. Pos: position; W: window; L: level.
The screening included usual blood tests, sputum and blood cultures, and urinary antigen tests for Pneumococcus and Legionella. The blood results showed a raised $\mathrm{C}$-reactive protein (CRP) level $\left(11.4 \mathrm{mg} \cdot \mathrm{dL}^{-1}\right.$; normal range $\left.<0.5 \mathrm{mg} \cdot \mathrm{dL}^{-1}\right)$, erythrocyte sedimentation rate (ESR) $\left(106 \mathrm{~mm} \cdot \mathrm{h}^{-1}\right.$; normal range $\left.<30 \mathrm{~mm} \cdot \mathrm{h}^{-1}\right)$ and lactate dehydrogenase level (674 IU. $\mathrm{L}^{-1}$; normal range 105-333 IU. $\left.\mathrm{L}^{-1}\right)$. The blood white cell count was normal (7520 cells. $\mu \mathrm{L}^{-1}$ with neutrophils count 5960 cells. $\left.\mu \mathrm{L}^{-1}(79.3 \%)\right)$ as was the platelets count (392000 platelets $\mu \mathrm{L}^{-1}$ ). Lower than normal values were noted for haematocrit (28.2\%) and haemoglobin $\left(9.33 \mathrm{~g} \cdot \mathrm{dL}^{-1}\right)$. The blood tests for liver function, urea, creatinine, electrolytes, albumin and collagen disease were normal. Repeated blood cultures and urinary antigen tests for Pneumococcus and Legionella were negative. Echocardiography showed normal dimensions and contractility, normal tricuspid aortic valve and absence of pericardial fluid.

The history of recent chemotherapy and the clinical signs suggested CAP as the first diagnostic choice. The patient was treated with intravenous antibiotics (moxifloxacine (Avelox) $400 \mathrm{mg}$ once per day). In addition, trimethoprim/sulphamethoxazole (Septrin) $20 \mathrm{mg} \cdot \mathrm{kg}^{-1} \cdot \mathrm{day}^{-1}$ was given in four equally divided doses, keeping in mind the probability of Pneumocystis jirovecii pneumonia in such an immunosuppressed patient. Oxygen was also delivered as supplementary.

Despite the applied antibiotic therapy, the patient's clinical status deteriorated. The hyperpyrexia continued. Dyspnoea and tachypnoea deteriorated (respiratory rate 40 breaths $\mathrm{min}^{-1}$, pulse oximetry $95 \%$ on $60 \%$ oxygen). Fast oxygen desaturation and talking difficulty was noted immediately after oxygen mask removal while platypnoea developed. Lung auscultation revealed dissemination of the crackles at all lung fields bilaterally.

On the third day of hospitalisation, a new chest radiograph showed similar findings to the initial chest radiograph. These ameliorated imaging findings were in unexplained controversy with the severe hypoxaemia and the auscultatory data. The patient underwent chest computed tomography (CT) with intravenous contrast material (figure $2 \mathrm{a}$ and $b$ ).

Task 4

Describe the chest CT findings. 


\section{Answer 4}

Chest CT showed diffuse bilateral ground-glass opacities affecting all lung fields, mainly the middle and lower ones (figure $2 \mathrm{~b}$ ), and bibasilar paraspinal consolidations. No findings of pulmonary embolism were observed.

\section{Task 5}

Based on the chest CT findings and the previous data, which is your diagnostic choice? 
Answer 5

Acute interstitial pneumonitis due to bleomycin-induced pulmonary toxicity.

Based on the recent chest CT findings along with the clinical deterioration under antibiotics, the clinical signs of bibasilar crackles, the normal blood cell count and the recent chemotherapy with bleomycin, acute pneumonitis due to bleomycin-induced pulmonary toxicity became the first diagnostic choice.

The patient was treated with intravenous prednisolone $1 \mathrm{mg} \cdot \mathrm{kg}^{-1}$ body weight with immediate dramatic improvement (remission of fever, decrease of dyspnoea and increase of oxygen saturation). The patient was discharged 7 days later without any symptoms and signs, with a respiratory rate of 18 breaths $\cdot \mathrm{min}^{-1}$, pulse oximetry $98 \%$ on room air, heart rate of 100 beats $\cdot \mathrm{min}^{-1}$, CRP of $0.317 \mathrm{mg} \cdot \mathrm{dL}^{-1}$ and ESR of $29 \mathrm{~mm} \cdot \mathrm{h}^{-1}$. On discharge, pulmonary function tests including forced vital capacity (FVC), forced expiratory volume in $1 \mathrm{~s}(\mathrm{FEV} 1), \mathrm{FEV}_{1} / \mathrm{FVC}$ ratio, single-breath diffusing capacity of the lung for carbon monoxide and transfer coefficient of the lung for carbon monoxide had values within normal predicted limits. Steroids were gradually tapered until stopping. 6 years later, the patient is in excellent condition.

In accordance with all the aforementioned data, the final diagnosis is acute pneumonitis due to bleomycin-induced pulmonary toxicity.

\section{Discussion}

This is a case of a young girl with Hodgkin lymphoma who developed severe bleomycininduced pneumonitis (BIP) [1].

Bleomycin is an antitumour agent isolated in 1966 from Streptomyces verticillus bacteria [2]. Its cytotoxic action is exerted primarily through DNA cleavage, resulting in cancer cell death. This process is dependent on iron ions and molecular oxygen [2, 3]. The enzyme bleomycin hydrolase, a cytosolic aminopeptidase, inactivates the cytotoxic effect of bleomycin [3].

Bleomycin is used for the treatment of various types of malignancies, such as both Hodgkin and non-Hodgkin lymphoma and germ cell tumours [2-4]. However, bleomycin chemotherapy has been associated with various toxicities, such as skin rashes, mucositis, hypersensitivity reactions and bleomycin pulmonary toxicity [5].

Pulmonary toxicity is the most important sideeffect during the therapeutic use of bleomycin. More specifically, the most common and severe pulmonary toxicity is BIP, which may progress to pulmonary fibrosis and is potentially lethal. Its incidence has been reported as $>39.8 \%$ [6] and mortality ranges from $1 \%$ [7] to $2.8 \%$ [5] of the patients treated with bleomycin. BIP fibrosis has been described in 6-10\% of cases receiving bleomycin [4]
The mechanism of BIP development is not fully clear. The low activity of bleomycin hydrolase enzyme in the lung is considered to be the main pathogenetic mechanism [3]. Oxidative damage, genetic susceptibility and release of inflammatory cytokines may play an additional role [2]. Additional risk factors have also been implicated in the development of BIP, such as older age (age $>40$ years), cumulative dose of bleomycin $>300$ IU, low glomerular filtration $\left(<80 \mathrm{~mL} \cdot \mathrm{min}^{-1}\right)$, advanced disease (stage IV disease at the beginning of bleomycin administration) [7], inhalation of high doses of oxygen, cigarette smoking and radiotherapy [2]. The mechanism of BIP development in the current case is not clear. We could not identify any specific risk factors in our patient. Genetic susceptibility remains questionable.

The disease is usually developed during treatment, as in the current case, but late presentation (several months after bleomycin chemotherapy) has also been reported [2, 4, 8]. BIP usually progresses gradually. In contrast, our patient developed acute rapidly progressive severe pneumonitis. The clinical presentation of the current case was not typical for BIP. Fever and dyspnoea, which were present in our patient, are usual symptoms of the disease, as are nonproductive cough and pleuritic pain [2, $4,8,9]$. Crackles were discovered first and there were characteristic findings on chest auscultation that supported the diagnosis of development of BIP [6].

BIP presents a variety of imaging patterns on chest radiograph and chest $C T$, obviously dependent on the course and severity of the disease. The imaging findings of the current case, although not typical of BIP, contributed to the diagnosis of the disease.

Radiographic manifestations may be absent in the early stages of the disease. They develop 1-2 weeks after the detection of crackles on chest ausultation [6]. Absence of radiographic manifestations in cases with severe pneumonitis has also been reported [9]. The typical radiographic findings of initial BIP stages are bilateral interstitial reticular or reticular micronodular lesions and alveolar infiltrates located mostly at the lower lung zones $[6,8]$. These lesions may progress to consolidations $[2,8]$ and further pulmonary fibrosis [2] or to remit until complete disappearance after treatment with steroids [8]. Bilateral involvement of lower lung fields as in the present case is a more typical finding during BIP. Nevertheless, asymmetric radiographic findings with involvement of only one lung have also been described [9].

Chest CT has been reported to be more sensitive than a chest radiograph for evaluating the pattern and distribution of lesions [10], and the present BIP case is in agreement. Various imaging patterns have been described on highresolution $\mathrm{CT}$ in patients with BIP: small linear 
and nodular subpleural lesions identified primarily in the posterior segments [11], pulmonary fibrosis with or without consolidation [10, 12], organizing pneumonia [12], nonspecific interstitial pneumonia [12], ground-glass opacities [10], widespread bilateral consolidation [10] and bronchial wall thickening with areas of decreased attenuation [10]. The mixed chest CT pattern of bilateral diffuse ground-glass and organizing pneumonia in our patient supported the diagnosis of a rather advanced stage of BIP.

The initial diagnosis of this case as CAP is a common diagnostic problem. BIP diagnosis is usually one of those remaining after exclusion of other possible diseases. A history of bleomycin chemotherapy and compatible clinical and radiological findings suggest the diagnosis of BIP, while transbronchial biopsy is not helpful in the diagnosis [2, 4, 6]. Besides CAP, pulmonary metastases or lymphangitic carcinoma may also be included in the differential diagnosis [2, 4].

There are no proven effective treatments for BIP. Bleomycin cessation and administration of steroids at high doses (prednisolone $1 \mathrm{mg} \cdot \mathrm{kg}^{-1}$ body weight, tapering down) is the standard treatment of BIP in clinical practice $[2,4,8,9]$.

The complete recovery of the current case with steroids reconfirms the full reversibility of the lesions in patients with BIP surviving the acute episode [2, 4, 8].

\section{Conclusions}

This is a case of a young girl with Hodgkin lymphoma who developed rapidly progressive BIP. The absence of risk factors demonstrates that the clinician should be suspicious of the development of pulmonary toxicity even in low-risk patients undergoing chemotherapy with bleomycin. Dyspnoea, cough, fever and crackles on chest auscultation are strong clinical signs of BIP development in patients under bleomycin treatment. Bilateral interstitial, alveolar and ground-glass opacities on high-resolution CT may help the diagnosis. CAP is the main feature in the differential diagnosis. Early recognition of BIP and initiation of steroid treatment is life saving and leads to full reversibility of the lesions.

\section{Conflict of interest}

None declared.

\section{References}

1. Haini E, Georgopoulos A, Haini D, et al. Bleomycin-induced pneumonitis in a 16-year-old girl with Hodgkin lymphoma and no risk factors: complete reversibility with steroids. Chest 2016; 149: Suppl., A190.

2. Sleijfer S. Bleomycin-induced pneumonitis. Chest 2001; 120 : 617-624.

3. Sikic BI. Biochemical and cellular determinants of bleomycin cytotoxicity. Cancer Surv 1986; 5: 81-91.

4. Rashid RS. Bleomycin lung: a case report. BMJ Case Rep 2009 [https://doi.org/10.1136/bcr.11.2008.1175].

5. Simpson AB, Paul J, Graham J, et al. Fatal bleomycin pulmonary toxicity in the west of Scotland 1991-95: a review of patients with germ cell tumours. BrJ Cancer 1998; 78: 1061-1066.

6. De Lena M, Guzzon A, Monfardini S, et al. Clinical, radiologic, and histopathologic studies on pulmonary toxicity induced by treatment with bleomycin (NSC-125066). Cancer Chemother Rep 1972; 56: 343-356.
7. O'Sullivan JM, Huddart RA, Norman AR, et al. Predicting the risk of bleomycin lung toxicity in patients with germ-cell tumours. Ann Oncol 2003; 14: 91-96.

8. Stein ME, Zidan J, Charas T, et al. Bleomycin-induced pneumonitis in three patients treated with chemotherapy for primary advanced seminoma. J BUON 2015; 20: 928-932.

9. White DA, Stover DE. Severe bleomycin-induced pneumonitis. Clinical features and response to corticosteroids. Chest 1984; 86: 723-728.

10. Padley SP, Adler B, Hansell DM, et al. High-resolution computed tomography of drug-induced lung disease. Clin Radiol 1992; 46: 232-236.

11. Rimmer MJ, Dixon AK, Flower CD, et al. Bleomycin lung: computed tomographic observations. BrJ Radiol 1985; 58: 1041-1045.

12. Usman $M$, Faruqui ZS, ud Din $N$, et al. Bleomycin induced pulmonary toxicity in patients with germ cell tumours. J Ayub Med Coll Abbottabad 2010; 22: 35-37. 